\title{
Modeling and Electromagnetic Scattering of Ground and Near Sea in Adjacent Region with Fractal Borderline
}

\author{
Gaoxiang Zou ${ }^{1+}$, Chuangming Tong ${ }^{1}$ Yalin Huang ${ }^{1}$ Hualong Sun ${ }^{1}$ Tong Wang ${ }^{1}$ \\ ${ }^{1}$ Air and Missile Defense College, Air Force Engineering University, Xi'an, China 710051
}

\begin{abstract}
A novel composite rough surface model is presented for describing ground and near sea in adjacent region. Monte Carlo method is used to generate composite rough surface. Gauss spectral function is used to simulate actual ground surface, and JONSWAP spectral function which is combined with shallow coefficient is used to simulate actual near sea surface. Weighted inverse tangent function is presented to connect two regions and smooth the borderline. One dimensional band-limited Weierstrass fractal function is introduced to simulate the actual borderline between ground and near sea in adjacent region. The geometrical models of composite rough surface with linear borderline and fractal borderline are given. By comparing of the contour maps, the geometrical model of composite rough surface with fractal borderline is closer to real environment of ground and near sea in adjacent region than the one with linear, borderline. The curves of bistatic scattering coefficient of ground, near sea and composite rough surfaces with linear and fractal borderline are compared. This paper presents a novel way to simulate the environment of ground and near sea in adjacent region. Simulation results can use for reference in the fields of remote sensing, detection and inversion of electromagnetic environment.
\end{abstract}

Keywords: ground and near sea in adjacent region, composite rough surface, weighted inverse tangent function, fractal borderline, electromagnetic scattering

\section{Introduction}

Nowadays, the electromagnetic scattering from the natural environment becomes one of the most important subjects in the field of computational electromagnetism and remote sensing[1-5]. Natural environment contains plain, mountain, hill, desert, valley and sea. Different methods for establishing geometrical model are proposed in order to simulate the natural environment and corresponding scattering characteristics of these environments are studied[6-10]. Monte Carlo method is often used to generate the rough surface. Different spectral functions are introduced to generate rough surfaces with different statistical properties, such as Gauss spectral function[6], exponential spectral function[7] and Pierson-Moskowitz spectral function[8]. However, these methods can only simulate the single environment precisely. Bare research has been reported on simulation of composite environment, especially the ground and near sea in adjacent region.

In this paper, a novel composite rough surface model is presented for describing ground and near sea in adjacent region. One dimensional band-limited Weierstrass fractal function is introduced to simulate the actual borderline between ground and near sea in adjacent region for the first time. Simulation results demonstrate that this method is feasible and efficient.

\section{Modeling of composite environment}

\subsection{Generation of composite rough surface}

\footnotetext{
Corresponding author. Tel.: +13072938219 .

E-mail address:gxiangzou@163.com.
} 
Gauss spectral function is used to simulate ground region. The parameters root mean square $h_{r m s}$ and correlation length $l_{x}, l_{y}$ can describe the statistical property of the ground rough surface. The concrete form of Gauss spectral function and the Monte Carlo process are involved in [9].

After generation of ground rough surface, we should find a precise way to generate the rough surface which is used to describe the statistical property of near sea surface. In [9], PM spectral function is firstly introduced to simulate the sea part of the ground and sea in adjacent region. But after our several investigations, we find out that the sea surface of ground and sea in adjacent region belongs to near sea range. Traditional PM spectral function[10] is a kind of omnidirectional sea spectrum which is often used to describe the abyssal sea area. Therefore, in consideration of effect of finite wind region, the wind region factor is introduced to transform the PM spectral function to JONSWAP spectral function[11]. Based on energy flow conservation theorem, the shallow coefficient and JONSWAP spectral function are combined into finite water depth spectral function[12] which is used to simulate actual near sea surface.

The PM spectral function is taken in the form:

$$
W_{P M}(k)=\frac{\alpha_{p}}{2 k^{3}} \exp \left(-\frac{\beta g^{2}}{U_{19.5}^{4} k^{2}}\right)
$$

Here $\alpha$ and $\beta$ are dimensionless empirical constants, $\alpha=8.10 \times 10^{-3}, \beta=0.74 . g_{c}$ is gravity acceleration, $g_{c}=9.81 \mathrm{~m} / \mathrm{s}^{2} . U_{19.5}$ is wind velocity on the height of $19.5 \mathrm{~m}$ above sea surface.

In actual sea surface, due to complexity of energy transformation among sea waves and polytropism of atmospheric conditions, sea surface is always under a unsteady state. In the late 1960s, based on the Joint North Sea Wave Project, JONSWAP sea spectrum was presented. JONSWAP sea spectrum is recognized as international standard sea spectrum. Based on PM spectral function, the JONSWAP spectral function is taken in the form:

$$
W_{\text {JONSWAP }}(k)=f \cdot W_{P M}(k)=f \cdot \frac{\alpha_{p}}{2 k^{3}} \exp \left(-\frac{\beta g^{2}}{U_{19.5}^{4} k^{2}}\right)
$$

Here $f$ is correction factor which is used to describe the effect of finite wind region, $f$ can be calculated using the expression:

Where

$$
f=\gamma^{B}
$$

$$
B=\exp \left\{-\frac{\left[\left(k / k_{p}\right)^{1 / 2}-1\right]^{2}}{2 \sigma_{J}^{2}}\right\}
$$

Here $k_{p}$ is wave number of dimensionless peak value, $k_{p}=g / U_{19.5}^{2} . \sigma_{J}$ is peak shape parameter which is defined as follow:

$$
\sigma_{J}=\left\{\begin{array}{l}
0.07, k \leq k_{p} \\
0.09, k>k_{p}
\end{array}\right.
$$

Here $\gamma$ is peak value enhancement factor, whose observed value ranges from 1.5 to 6 and mean value is 3.3. Brüning and others present that the expression (3) can simulate spectral function with different sea scale by different values of $\gamma$ and $\alpha_{p}$ [13]. when $\alpha_{p}=0.01, \gamma=3.3$, it is unformed JONSWAP spectrum.

Unformed JONSWAP spectrum is used to describe near sea surface. However, near sea located in ground and near sea in adjacent region is in shallow water of finite depth. The influence of finite depth must be taken into consideration. Thus, we introduce shallowing coefficient [14] to combine with JONSWAP spectral function into finite water depth spectral function in order to simulate the sea surface which located in ground and near sea in adjacent region. The finite water depth spectral function is taken in the form:

$$
W_{\text {finite }}(k, d)=\zeta^{2}(d) \cdot W_{\text {JONSWAP }}(k)
$$

Shallowing coefficient is defined as radio of wave height at finite water depth $d$ to wave height at infinite water depth which is deduced according to energy flow conservation theorem[12]. It can be calculated using the expression:

$$
\zeta(d)=\sqrt{\frac{\cosh ^{2}(k d)}{\sinh (k d) \cosh (k d)+k d}}
$$

With the revision of shallowing coefficient, the modeling of ground and near sea in adjacent region will be closer to the actual environment. 
According to the International Maritime Bureau, water depth ranges from 50 to $600 \mathrm{~m}$ which belongs to near sea region. The water depth in the range of continental shelf is often less than $200 \mathrm{~m}$. The slope of seabed is about ranging from 0 to 0.9. On the above basis, an approximate method is presented in Fig. 1 to simulate the water depth in the range of continental shelf. This method is just a simple and approximate way to deal with the problem of water depth simulation. If we can obtain the datum of actual water depth in the range of near sea, the water depth model would be more accurate and reasonable.

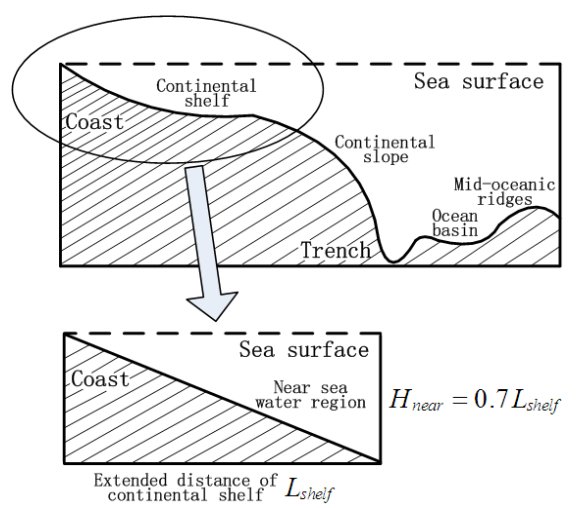

Fig.1: Approximate method of water depth simulation

\subsection{Generation of fractal borderline}

One dimensional band-limited Weierstrass fractal function [15] is introduced to simulate the actual borderline between ground and near sea in adjacent region. One dimensional band-limited WeierstrassMandelbrot fractal function is derived from Weierstrass function, which can keep main fractal characteristic because this function has internal and external scale in some degree. Band-limited Weierstrass fractal function is taken in the form:

$$
f_{\text {fractal }}(x)=\sqrt{2} R \sqrt{\frac{1-b^{2(D-2)}}{1-b^{2 N(D-2)}}} \sum_{n=0}^{N-1} b^{n(D-2)} \cos \left(k_{0} b^{n} x+\varphi_{n}\right)
$$

Here $R$ is used to control fluctuation intensity of the borderline. $b$ is scale factor. $D$ is fractal dimension. $N$ is the highest harmonic wave number. $k_{0}$ is spatial wave number which determine the position of spatial frequency spectrum. $\varphi_{n}$ is random phase which is distributed evenly in $(0,2 \pi)$. The highest harmonic wave number can be confirmed by incident wave frequency. It can be calculated using the expression: $k_{0}=k / b^{N-1}$. Here $k$ is incident wave number. This expression can ensure that the highest harmonic wave number is less than incident wave length. In the paper, we use one dimensional band-limited Weierstrass fractal function to simulate the actual borderline between ground and near sea in adjacent region.

\section{Simulation results}

The borderline of every partial area which belongs to ground and near sea in adjacent region can be simplified into linear borderline. The weighted inverse tangent function with linear borderline is taken in the form:

$$
f_{\text {com }}(x, y)=\left[\pi / 2-\arctan \left(y-y_{b}\right)\right] / \pi \cdot f_{\text {gro }}(x, y)+\left[\pi / 2+\arctan \left(y-y_{b}\right)\right] / \pi \cdot f_{\text {sea }}(x, y)
$$

Here $f_{g r o}(x, y)$ and $f_{\text {sea }}(x, y)$ present height profiles of ground rough surface and near sea surface respectively. $y_{b}$ represents position of borderline, it can also be took as $x_{b}$ position according to actual environment. The sampling of the incident frequency is $f=300 \mathrm{MHz}$. The size of composite rough surface is $40 \lambda \times 40 \lambda$. Statistic parameters of ground region are: $h_{r m s}=1.0 \lambda, l_{x}=l_{y}=1.0 \lambda$. Statistic parameters of near sea region are: $U_{19.5}=5 \mathrm{~m} / \mathrm{s}$, wind range $X=50 \mathrm{~km}$. Shallowing coefficient is calculated based on Fig. 1 . The parameter of borderline is $y_{b}=0$. Five sampling points are considered per wave length. The composite rough surface model with linear borderline is obtained as shown in Fig.2. 


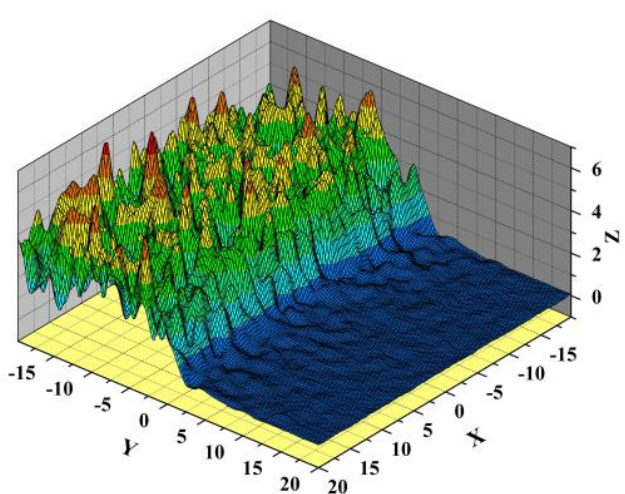

Fig. 2: Composite rough surface with linear borderline

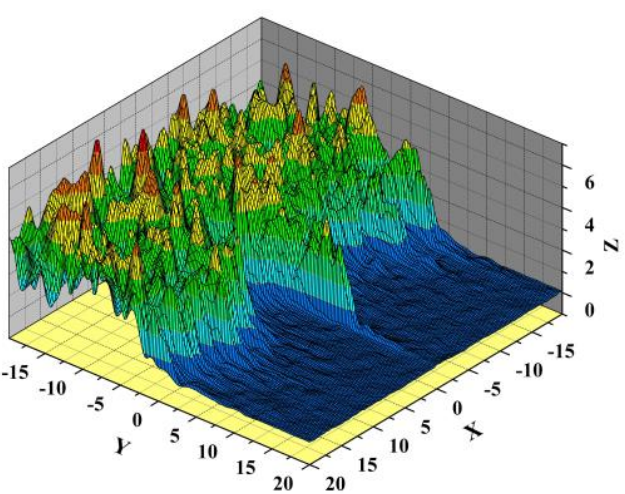

Fig. 3: Composite rough surface with fractal borderline

One dimensional band-limited Weierstrass fractal function is introduced to elaborate the borderline. The weighted inverse tangent function with fractal borderline is taken in the form:

$$
f_{\text {com-fractal }}(x, y)=\left[\pi / 2-\arctan \left(y-y_{b}+f_{\text {fractal }}(x)\right)\right] / \pi \cdot f_{\text {gro }}(x, y)+\left[\pi / 2+\arctan \left(y-y_{b}+f_{\text {fractal }}(x)\right)\right] / \pi \cdot f_{\text {sea }}(x, y)
$$

Here $f_{\text {fractal }}(x)$ is profile of fractal borderline. Statistic parameters of fractal borderline are: $R=25 \lambda, b=0.5 e$ and $D=1.5$. The composite rough surface model with fractal borderline is obtained as shown in Fig.2.

In Fig.2 and Fig.3, both of them realize geometrical modeling of ground and near sea in adjacent region. Obviously, the shape of borderline in Fig. 2 is the simplest way to structure the composite rough surface model. By contrast, the fractal borderline in Fig. 3 is similar to the natural borderline. The contour maps of composite rough surfaces above are given in Fig.4.
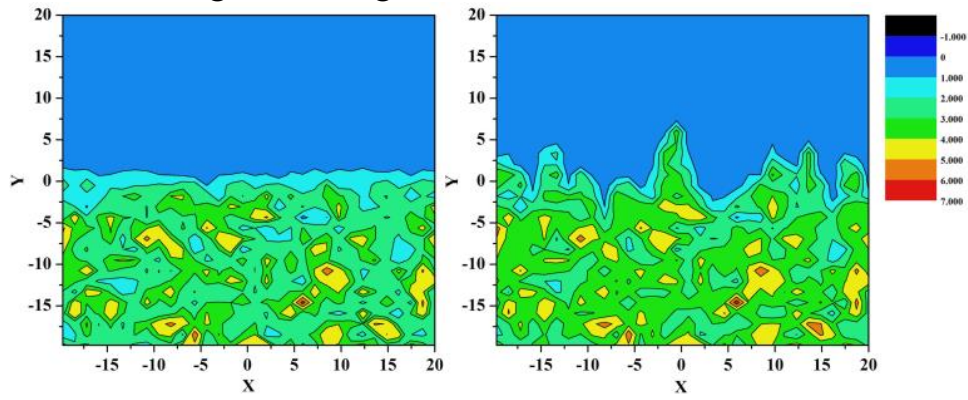

Fig. 4: Contour maps of composite rough surface with linear and fractal borderline

In Fig.4, the transitional zone is obviously observed between ground and near sea. By comparing of the contour maps, the geometrical model of composite rough surface with fractal borderline is closer to real environment of ground and near sea in adjacent region than the one with linear borderline.

Based on the geometrical models above, the differences of scattering characteristics of single ground rough surface, single sea rough surface and composite rough surface with linear or fractal borderline are compared by IPO method which is based on the iteration of electric and magnetic current[9]. The incident frequency is $f=300 \mathrm{MHz}$. Incident angles are: $\theta_{i}=45^{\circ}, \varphi_{i}=0^{\circ}$. Polarization mode is HH polarization. The size of all rough surfaces is $40 \lambda \times 40 \lambda$. Statistic parameters of ground rough surface are: $h_{r m s}=1.0 \lambda$, $l_{x}=l_{y}=1.0 \lambda$. Relative dielectric constant of ground region is $(7.0,-2.4 \mathrm{i})$. Statistic parameters of near sea rough surface are: $U_{19.5}=5 \mathrm{~m} / \mathrm{s}$, wind range $X=50 \mathrm{~km}$. Shallowing coefficient is calculated based on Fig. 1 . Relative dielectric constant of near sea region is (20.0,-240i). Statistic parameters of ground region and near sea region are the same as above. Five sampling points are considered per wave length. We build 50 samples of every kind of rough surface and calculate every sample by IPO. Final results are obtained by taking the average of results of these samples. 


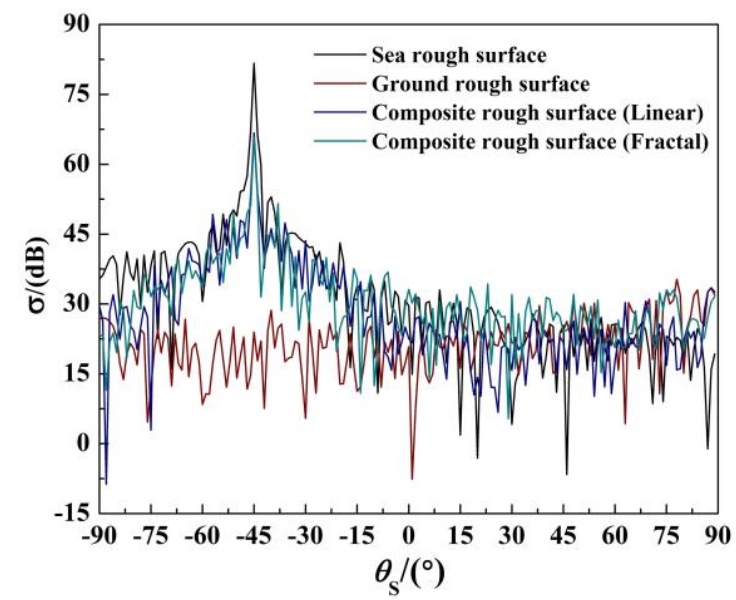

Fig.5: The bistatic scattering coefficients of different rough surface models

In Fig.5, the bistatic scattering coefficient (BSC) of ground rough surface presents a characteristic of diffuse scattering. The BSC of near sea rough surface presents a characteristic of intense specular scattering. We can find interesting results of composite rough surface from BSCs. The electromagnetic scattering energy distributes dispersedly in region far from direction of specular reflection, but the electromagnetic scattering energy concentrates on the region near direction of specular reflection. As a result, composite rough surface not only has the electromagnetic scattering characteristic of ground rough surface, but also has the electromagnetic scattering characteristic of sea rough surface. By contrast, the BSC of composite rough surface with linear borderline is similar as the one with fractal borderline. Both of them have a peak value near the direction of specular reflection. But in aspect of the diffuse scattering in other angles, composite rough surface with fractal borderline is stronger than the one with linear borderline. The complex construction of fractal borderline will lead to more coupling times between the facets of ground rough surface and sea rough surface. Therefore, composite rough surface with fractal borderline presents a characteristic of diffuse scattering in region far from direction of specular reflection.

\section{Conclusions}

In this paper, a novel composite rough surface model is presented for describing ground and near sea in adjacent region. One dimensional band-limited Weierstrass fractal function is introduced to simulate the actual borderline between ground and near sea in adjacent region for the first time. Simulation results demonstrate that this method is feasible and efficient. The further works are taking the target into consideration to study the composite scattering characteristics of targets and composite rough surface.

\section{References}

[1] H. Zamani, A. Tavakoli, M. Dehmollaian. Second-Order Perturbative Solution of Cross-Polarized Scattering From Multilayered Rough Surfaces. IEEE Transactions on Antennas and Propagation, 2016, 64(5):1877-1890.

[2] R. Wang, L. Guo, Z. Zhang. Scattering From Contaminated Rough Sea Surface by Iterative Physical Optics Model. IEEE Geoscience and Remote Sensing Letters, 2016, 13(4):500-504.

[3] Z. H. Lai, J. F. Kiang, R. Mittra. A Domain Decomposition Finite Difference Time Domain (FDTD) Method for Scattering Problem from Very Large Rough Surfaces. IEEE Transactions on Antennas and Propagation, 2015, 63(10):4468-4476.

[4] J. Li, M. Zhang, P. Wei, et al. An Improvement on SSA Method for EM Scattering From Electrically Large Rough Sea Surface. IEEE Geoscience and Remote Sensing Letters, 2016, 13(8):1-5.

[5] A. Montuori, F. Nunziata, M. Migliaccio, et al. X-Band Two-Scale Sea Surface Scattering Model to Predict the Contrast due to an Oil slick. IEEE Journal of Selected Topics in Applied Earth Observations and Remote Sensing, 2016, 9(11):4970-4978.

[6] A. G. Voronovich, V. U. Zavorotny. Full-Polarization modeling of monostatic and bistatic radar scattering from a rough sea surface. IEEE Transactions on Antennas and Propagation, 2014, 62(3):1362-1371. 
[7] A. Darawankul, J. T. Johnson. Band-Limited exponential correlation function for rough-surface scattering. IEEE Transactions on Geoscience and Remote Sensing, 2007, 45(5):1198-1206.

[8] R. Wang, L. X. Guo, A. Q. Wang. Study on the scattering from sea surface with PM spectrum covered by organic film. IEEE International Symposium on Antennas Propagation and EM Theory, 2010, pp:738-741.

[9] G. X. Zou, C. M. Tong, T. Wang, H. L. Sun. Electromagnetic scattering characteristics of composite random rough surface of adjacent region between ground and ocean on Terahertz waveband. IEEE MTT-S International Microwave Workshop Series on Advanced Materials and Processes for RF and THz Applications, 2016:1-4.

[10] W. J. Pierson, L. Moscowitz. A proposed spectral form for fully developed wind seas based on the similarity theory of S.A.kitaigorodsrii. J. Geophys. Res., 1964, 69(24): 5181-5190.

[11] K. Hasselmann, T. P. Barnett, E. Bouws, et al. Measurements of wind-wave growth and swell decay during the Joint North Sea Wave Project(JONSWAP). Dtsch. Hydrogr. Z. Suppl., 1973, 12(A8):1-95.

[12] D. Nie, M. Zhang, C. Wang, H. C. Yin. Study of microwave backscattering from two-dimensional nonlinear surfaces of finite-depth seas. IEEE Transactions on Geoscience and Remote Sensing, 2012, 50(11): 4349-4357.

[13] C. Brüning, W. Alpers, K. Hasselmann. Monte-carlo simulation studies of the nonlinear imaging of two dimensional surface wave field by a synthetic aperture radar. International Journal of Remotes Sensing, 1990, 45(10): 487-494.

[14] M. E. Mccormick. Ocean engineering wave mechanics. New York: John Wiley \& Sons Inc, 1973.

[15] X. M. Zhu, X. C. Ren, L. X. Guo. FDTD investigation on electromagnetic scattering of 1D band-limited Weierstrass fractal land surface and a conducting column with rectangular cross-section. Chinese Journal of Computational Physics, 2012, 29(3):399-405. 